\title{
From Relational ClassSheets to UML+OCL
}

\author{
Jácome Cunha* \\ Universidade do Minho \\ Portugal \\ jacome@di.uminho.pt
}

\author{
João Paulo Fernandes \\ Universidade do Minho \& \\ Universidade do Porto \\ Portugal \\ jpaulo@di.uminho.pt
}

\author{
João Saraiva \\ Universidade do Minho \\ Portugal \\ jas@di.uminho.pt
}

\begin{abstract}
Spreadsheets are among the most popular programming languages in the world. Unfortunately, spreadsheet systems were not tailored from scratch with modern programming language features that guarantee, as much as possible, program correctness. As a consequence, spreadsheets are populated with unacceptable amounts of errors.

In other programming language settings, model-based approaches have been proposed to increase productivity and program effectiveness. Within spreadsheets, this approach has also been followed, namely by ClassSheets. In this paper, we propose an extension to ClassSheets to allow the specification of spreadsheets that can be viewed as relational databases. Moreover, we present a transformation from ClassSheet models to UML class diagrams enriched with OCL constraints. This brings to the spreadsheet realm the entire paraphernalia of model validation techniques that are available for UML.
\end{abstract}

\section{Categories and Subject Descriptors}

D.2.4 [Software Engineering]: Software/Program Verification; D.2.2 [Software Engineering]: Object-oriented design methods; F.1.1 [Models of Computation]: [Relations between models]

\section{General Terms}

Design, Languages, Verification

\section{Keywords}

Spreadsheets, UML, OCL, ClassSheets ${ }^{*}$ Supported by Fundação para a Ciência e a Tecnologia,
grant SFRH/BPD/73358/2010.
${ }^{\dagger}$ Supported by Fundação para a Ciência e a Tecnologia,
grant SFRH/BPD/46987/2008.
Work supported by the SSaaPP project, FCT contract

PTDC/EIA-CCD/108613/2008.

Permission to make digital or hard copies of all or part of this work for personal or classroom use is granted without fee provided that copies are not made or distributed for profit or commercial advantage and that copies bear this notice and the full citation on the first page. To copy otherwise, to republish, to post on servers or to redistribute to lists, requires prior specific permission and/or a fee.

SAC'12 March 25-29, 2012, Riva del Garda, Italy.

Copyright 2011 ACM 978-1-4503-0857-1/12/03 ...\$10.00.

\section{INTRODUCTION}

Spreadsheets are widely used by non-professional programmers, the so-called end users, to develop business applications. Spreadsheet systems offer a high level of flexibility, making it easy to start working with them. This freedom, however, comes with a price: spreadsheets are error prone as shown by numerous studies which report that up to $90 \%$ of real-world spreadsheets contain errors $[12,13,14]$.

In recent years the spreadsheet research community has recognized the need to support end-user model-driven software development, and to provide spreadsheet developers and end users with methodologies, techniques and the necessary tool support to improve their productivity. Along these lines, several techniques have been proposed [1, 6, 9], being ClassSheets [7] the most powerful domain specific model for spreadsheets. ClassSheets provide a powerful paradigm for model-driven software development: a spreadsheet business model is first defined, from which a customized spreadsheet application is generated guarantying the consistency of the spreadsheet with the underlying model.

Despite of its huge benefits, the ClassSheets model has two important drawbacks: firstly, it is not powerful enough to capture common end user errors. For example, a great number of spreadsheets represent database tables ${ }^{1}$, where there is an embedded notion of a table key in one column (as known from database theory). In this context, end users should be warned by the spreadsheet system if, e.g., a duplicated key is introduced in such a column. In fact, in [6] we have proposed techniques for spreadsheet edit assistance that rely on relational database models, and that guide end users in introducing correct data. The ClassSheet model is a very table syntactic oriented formalism and it lacks this notion of relational databases [10].

Secondly, although ClassSheets is a model-based formalism, there is no connection between it and the languages and techniques developed by the modelware community. In fact, the modelware community has done a considerable amount of work on model design, transformation, evolution and coevolution of models and instances [2], that we are convinced the ClassSheet formalism could reuse and benefit from.

The goal of this paper is three-fold:

- Firstly, we extend ClassSheets with the notion of relational databases. As a result, the spreadsheet data is not only organized in ClassSheets tables, but such tables have also the notion of primary and foreign keys.

\footnotetext{
${ }^{1}$ Studies suggest that a huge percentage of spreadsheets are in fact databases, since no formula is used in them! [3].
} 
- Secondly, we extend our embedding of the ClassSheets in a spreadsheet system [5] to support the so-called relational ClassSheets. We also extend our embedding so that it produces customized spreadsheets that guides end user to edit data that conforms to such relational ClassSheets.

- Thirdly, we present a transformation from relational ClassSheets to UML+OCL, and thus, making all paraphernalia of techniques for model validation, transformation and evolution available to relational ClassSheets.

The rest of the paper is organized as follows: in Section 2 we review the ClassSheet modelling language, which is extended with notions from relational databases in Section 3. Section 4 presents a transformation from this extended modelling language to UML classes with OCL constraints. In Section 5 we compare ours and related works and in Section 6 we conclude the paper.

\section{CLASSSHEETS}

ClassSheets [7] are a high-level, object-oriented formalism to specify the business logic of spreadsheets. ClassSheets allow users to express business object structures within a spreadsheet using concepts from the Unified Modeling Language (UML). Using the ClassSheets model, it is possible to define spreadsheet tables and give them names, define labels for the table's columns, specify the types of the values such columns may contain and also the way the tables expand (e.g., horizontally or vertically).

In this section we review the embedding of ClassSheets in spreadsheet systems, a technique that we have proposed in [5]. We mimic the well-known embedding of a domain specific language in a general purpose one and inherit all the powerful features of the host language: in our case, the powerful interactive interface offered by the (host) spreadsheet system. In order to present our embedding, we consider a model-based spreadsheet system, adapted from [10], to manage an airline company. The fragment of the ClassSheet model for such a system that deals with the pilots' salaries is given in Figure 1.

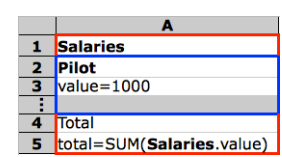

Figure 1: Using ClassSheets to create a Salaries sheet.

From an object-oriented point of view, one can see a summation object, which aggregates a list of objects containing single values. Looking at the layout structure, and starting from the blue part ${ }^{2}$, we see a class labeled Pilot, consisting of a value for which the default is 1000 . In fact, this is not a single value, but a list of values, since the row after it is labeled with ellipses. The summation object, in red, is defined by a label Salaries, a footer labeled Total and an aggregation formula assigned to an attribute named total.

Such an object-oriented extended template is a ClassSheets model since it defines classes together with their attributes and aggregational relationships. ClassSheets consist of a list of attribute definitions grouped by classes and

\footnotetext{
${ }^{2}$ We assume the digital version of the paper to be colored.
}

arranged on a two dimensional grid. Additional labels are used to annotate the concrete representation. References to other entries are defined by using attribute names, as shown in the SUM formula in the example. The formal language of ClassSheets is as follows [7]:

$$
\begin{array}{lll}
f \in F m l & :=\varphi|n . a| \varphi(f, \ldots, f) & \text { (formulas) } \\
b \in \text { Block } & ::=\varphi|a=f| b|b| b^{\wedge} b & \text { (blocks) } \\
l \in \text { Lab } & ::=h|v| . n & \text { (classlabels) } \\
h \in \text { Hor } & ::=\underline{n}|| n & \text { (horizontal) } \\
v \in \text { Ver } & ::=|n| \frac{n}{\mid} & \text { (vertical) } \\
c \in \text { Class } & ::=l: b\left|l: b^{\downarrow}\right| c^{\wedge} c & \text { (classes) } \\
s \in \text { Sheet } & ::=c\left|c^{\rightarrow}\right| s \mid s & \text { (sheets) }
\end{array}
$$

In the next sections, we go through several concrete illustrations of constructions that are common to both spreadsheet systems and ClassSheets.

\subsection{Vertically Expandable Tables}

Suppose that we want to record the activity of the company's pilots. A simple way of achieving this is to use a spreadsheet, and a table as the one presented in Figure 2a. This table has a title, Pilots, and a row with labels, one for each of the table's column: ID represents a unique pilot identifier, Name represents the pilot name and Flight hours represents the total number of hours a pilot has flown. Each of the subsequent rows represents a concrete pilot.

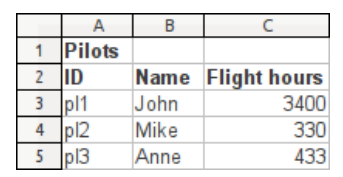

(a) A concrete table.

Pilots : Pilots

Pilots : ID

Pilots : $\quad(\mathrm{id}=$ " "

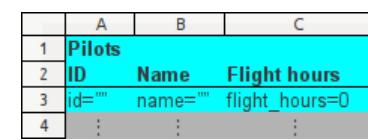

(b) The visual model.

$\begin{array}{lll}\sqcup & \text { | } & \sqcup^{\wedge} \\ \text { Name } & \text { | } & \text { Flight hours } \\ \text { name= " " } & \text { | } & \text { flight_hours=0) }\end{array}$

(c) The formal model.

Figure 2: Pilot activity record.

Tables such as the one presented in Figure 2a are frequently used within spreadsheets, and it is fairly simple to create a model specifying such tables. For the example shown, we can extract the visual ClassSheets model presented in Figure 2b and its corresponding formal definition shown in Figure 2c.

To model the labels we use a textual representation and the exact same names as in the data sheet (Pilots, ID, Name and Flight hours). To model the actual data we abstract concrete column cell values by using a single identifier: we use the one-worded, lower-case equivalent of the corresponding column label (so, id, name and flight_hours). Next, a type is associated with each column: columns A and $\mathrm{B}$ hold strings and column $\mathrm{C}$ holds integer values (denoted in the model, respectively, by the empty string "' and 0 following the = sign). Notice that the fourth row of the visual model contains vertical ellipses in all columns. This means that it is possible for this column to expand vertically: the tables that conform to this model can have has many rows as needed. The scope of the expansion is between the ellipsis and the black line (between row 2 and 3 ). In the formal model this expansion possibility is expressed by the $\downarrow$ sign which affects the same spreadsheet elements.

\subsection{Horizontally Expandable Tables}


An airline company must also store information on its airplanes. This is the purpose of table Planes in the spreadsheet illustrated in Figure 3a, which is organized as follows: the first column holds labels that identify each row, namely, N-Number, Model and Name; cells in row N-Number (respectively Model and Name) contain the unique identifier of a plane, (respectively the model of the plane and the name of the plane). Each of the subsequent columns contains information about one particular aircraft.

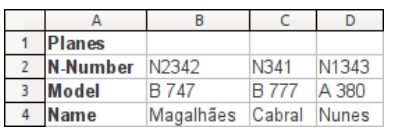

(a) A concrete table.

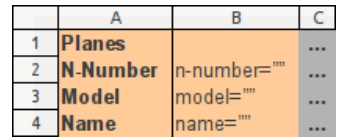

(b) The visual model.

$\sqcup$
n-number $=" "$
model $=" "$
name $=" ") \rightarrow$

(c) The formal model.

Figure 3: Information on airplanes.

The Planes table can be modelled by the illustration in Figure $3 \mathrm{~b}$, whose formal definition is given in Figure 3c. This model may be constructed following the same strategy as in the previous section, but now swapping columns and rows. The first column contains the label information and the second column the names abstracting concrete data values; again, each cell has a name and the type of the elements in that row (in this example, all cells are to hold strings). The third column has ellipses meaning that rows are horizontally expandable. Notice that the instance table has information about three planes.

\subsection{Relationship Tables}

So far in the paper, we have modelled tables for pilots and planes; reusing what we built, we can now model, as shown in Figure 4a, a table to store information on concrete flights.

We can see some of the information we had before: in the top left corner we have a concrete spreadsheet where, between rows 8 and 12, we have the pilot information shown in Figure $2 \mathrm{a}$ and between rows 15 and 18 the plane information shown in Figure 3a. The lines between rows 1 and 5 represent flight scheduling information. For simplicity, let us for now focus on columns A to E. Column A holds the identifier of the pilot for a concrete flight. Row 2, columns $\mathrm{B}$ and $\mathrm{F}$, hold the identifiers of the airplanes assigned to fly from $O P O$ to $N A T$, two times, and from LIS to AMS, respectively. Origins and destinations of flights are registered in Depart and Destination columns, as well as the date and hour of departure (column Date) and the number of hours the flight will take (column Hours). Notice that we can have as many entries for pilots (planes, respectively) as we need just by adding one row per pilot (and 4 columns per plane). An example of how we read this table is as follows: pilot pl1 flew plane N2342 from OPO to NAT on December 12th, 2010, at 14:00 hours and the flight took 7 hours.

The ClassSheet model illustrated in the bottom-right part of Figure 4a straightforwardly abstracts the data instance that we have just described. The top block of cells expand both vertically and horizontally as indicated by the ellipses.
The vertical expansion is necessary to add more pilots; the horizontal one to add more planes. The colors in the model are used to distinguish the different entities represented, namely, pilots, planes, references to pilots in the scheduling table, reference to planes in the scheduling table and the flight scheduling itself. In the formal syntax of ClassSheets, the visual model is represented as shown in Figure 4b. Due to space constraints, this representation has been given a vertical look, but actually the model is horizontally composed by three blocks (separated by ।).

\subsection{Generating Spreadsheets from ClassSheets}

The previously described models can be translated into initial spreadsheets together with tailor-made versions of update operations. These operations are defined to perform the tasks of insertion or deletion in such a way that the spreadsheet correctness is always preserved. The model presented in the bottom-right part of Figure 4a can be used to generate the spreadsheet in the top-left part of the same figure that guides end-users introducing correct data (actually, this spreadsheet has already been edited after the initial generation). The generated spreadsheet contains the labels in bold on the model, the initial formulas and buttons to add new vertical and horizontal blocks of cells. For example, in the Pilots table, there is a button on row 13 which will insert a new row. The values that will appear in the new row are the default values defined in the model and the user can only update them to a value of the same type (string, integer, etc.). A more complex situation would be to add a new flight; this involves a pilot and a plane, and some more information. If the user clicks the button in row 6 , the system will add a new row as explained before, but in this case it will also update the necessary formulas: it will update the formulas in cells E7, I7 and K7 to include the new added row. This mechanism prevents the user from editing the spreadsheet without correctly updating its formulas, and therefore from corrupting it. The button in column $\mathrm{J}$ works in a similar way, updating the formulas in cells $\mathrm{K} 4$ and $\mathrm{K} 5$.

\section{CLASSSHEETS AND DATABASES}

In the previous section we have reviewed ClassSheets as proposed in [7] and we have shown a concrete example of how it can be used in practice. In this section we extend the original ClassSheet formal language with constructions from the relational database realm. In this way, we extend further the amount of validations that are possible for spreadsheets derived from ClassSheets.

We propose the following extensions, marked in red:

$$
\begin{array}{lll}
f \in F m l & ::=\varphi|n . a| \varphi(f, \ldots, f) & \text { (formulas) } \\
b \in \text { Block } & ::=\varphi\left|\varphi^{\mu}\right| a=f|b| b \mid b^{\wedge} b & \text { (blocks) }
\end{array}
$$

In a model, as a consequence of the syntactic extension $\varphi^{\mu}$, a column or row can now be declared as containing unique values. Each of these values is thought of as a primary key in a relational database. This has important semantic consequences. Consider, for example, the pilot activity record presented in Section 2.1. There, we declared that the ID column should hold strings, but the model was not rich enough to ensure that each pilot in the derived spreadsheet (or each row in the corresponding column) has a unique identification. This can cause two different problems/errors: 1) two different pilots could be given the same ID, with obvious implications (one being, for example, a pilot assigned to fly a 


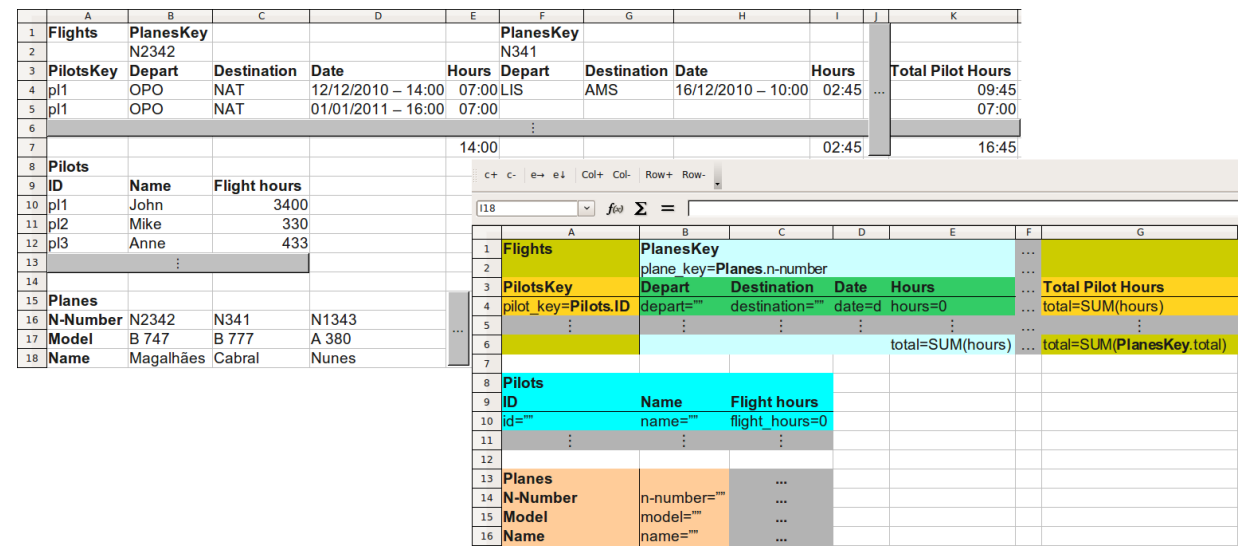

(a) Spreadsheet instance and ClassSheet model of an airline company.

\begin{tabular}{|c|c|}
\hline $\begin{array}{l}\text { Flights : } \\
\text { Flights : } \\
\text { PilotsKey : } \\
\text { PilotsKey : }\end{array}$ & $\begin{array}{l}\text { Flights } \\
\sqcup \\
\text { PilotsKey } \\
\text { (pilot_key = Pilots.id) })^{\downarrow}\end{array}$ \\
\hline $\begin{array}{l}\text { Planeskey : } \\
\text { Planeskey : } \\
\text { Flight : } \\
\text { Flight : } \\
\text { Planeskey : }\end{array}$ & $\begin{array}{l}\text { Planeskey } \\
\text { plane_key=Planes.n-number } \\
\text { (Depart । Destination । Date । Hours } \\
(\text { depart }=" \text { " । destination }=" \text { " । date }=\mathrm{d} \text { । hours }=0)^{\downarrow} \\
\sqcup । \sqcup|\sqcup| \text { total }=\operatorname{SUM}(\text { hours })) \rightarrow\end{array}$ \\
\hline $\begin{array}{l}\mid \text { Flights : } \\
\mid \text { Flights : } \\
\text { TotalPilotHours : } \\
\text { |TotalPilotHours : } \\
\text { |Flights : }\end{array}$ & $\begin{array}{l}\text { Flights } \\
\text { Flights } \\
\text { TotalPilotHours } \\
(\text { total=SUM(hours }))^{\downarrow} \\
\text { total = SUM(PlanesKey.total })\end{array}$ \\
\hline
\end{tabular}

(b) The formal model.

Figure 4: Spreadsheet of an airline company and an abstract model representing it.

co-worker's flight); 2) the same pilot could occur in different rows, with potentially different flight hour records.

This extension is useful for the models that we have seen so far. In fact, it should be used for the fragments storing both the information on pilots and planes of Figures 2 and 3, whose identifiers must be unique:

\begin{tabular}{|c|c|c|c|c|c|c|c|}
\hline \multirow{3}{*}{$\begin{array}{l}\text { Pilots : } \\
\text { Pilots : } \\
\text { Pilots : }\end{array}$} & \multirow{2}{*}{$\begin{array}{l}\text { Pilots } \\
{ }^{\mu}\end{array}$} & । & & & \multirow{3}{*}{\multicolumn{2}{|c|}{$\begin{array}{l}\sqcup^{\wedge} \\
\text { Flight Hours } \\
\text { flight_hours }=0)^{\downarrow}\end{array}$}} \\
\hline & & 1 & Name & & & & \\
\hline & $(\mathrm{id}=\mathrm{"} "$ & । & name $=$ & " " & & & \\
\hline Planes & & Plan & & 1 & ᄂ & & 个 \\
\hline N-Numbe & & $(\mathrm{N}-\mathrm{N}$ & amber $^{\mu}$ & 1 & & umber- & ^ \\
\hline Model : & & Mod & & 1 & & del= " " & \\
\hline Name & & $\mathrm{Nam}$ & & 1 & & $\mathrm{ne}=\mathrm{n} " \mathrm{n}) \rightarrow$ & \\
\hline
\end{tabular}

Now, and just as an example of the extension at work, if a user tries to insert a new pilot with an identifier already in use, the spreadsheet derived from the pilots model above is now able of producing the warning show in Figure 5.

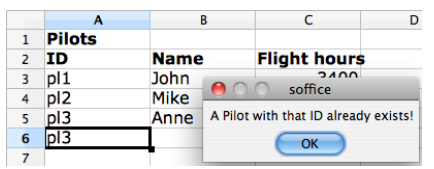

Figure 5: Uniqueness value validation.

As for the extension n.a, it is actually not of a syntac- tic nature. Indeed, you may notice that such a definition already occurred in the original ClassSheet language as a way of referring, in a class, to an attribute of a different class. In our work, however, this has a stronger interpretation. Indeed, we view one such occurrence in the same way a foreign key is viewed in a database model. This means that, when a spreadsheet model is being constructed, we first check whether a class $n$, and the corresponding attribute $a$ do exist before accepting a cell declaration n.a. Then, we also verify whether $a$ has been declared as unique (i.e., as a primary key) in block $n$. A final validation of the derived spreadsheet is ensured by construction: it is not possible to have erroneous values in these cells since the user is only allowed to fill them by selecting a value from a list with precisely the values in n.a. Actually, in case the user inserts a value of the appropriate type in a foreign key cell, we do not immediately consider an error the fact that such value is not a primary key of the corresponding table: a situation like that may reveal an intention of introducing a new tuple (key, values) in that table. Therefore, the user is in this case asked either to confirm the erroneous situation or to introduce the values to associate with key, that we correctly insert in the right table. A concrete situation of this kind is illustrated in Figure 6, when the user tries to assign pilot $p l 4$ to a given flight whereas no pilot with that PilotsKey exists in the Pilots table.

These validations were originally not ensured and, at the 


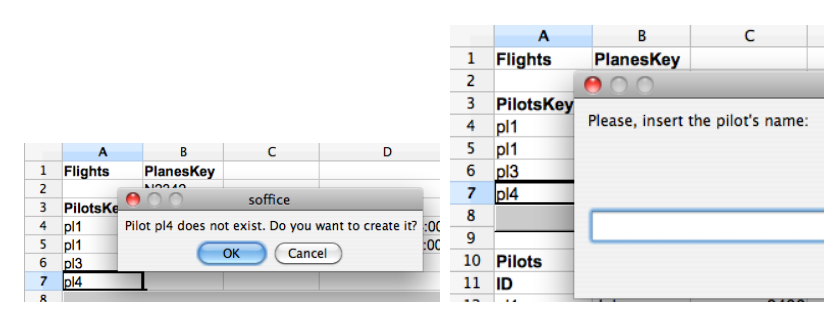

Figure 6: Foreign key validation.

model level, we could easily point (for example, as a result of miss-typing) to a non existing attribute. Also, at the spreadsheet level, there was no guarantee that the concrete value inserted in the spreadsheet was or not correct.

Not being syntactic, this extension would not require any change in the models presented in the previous section. However, it is by the new semantic interpretation of Pilots.id, Planes.n-number and PlanesKey.total that these references are guaranteed to be correct in all the different lines just described.

Technically, these validations are ensured, either at the (embedded) model level or at the spreadsheet level, by a series of scripts in the BASIC scripting language and under the spreadsheet system from the OpenOffice.org [11] suite.

\section{FROM CLASSSHEETS TO UML+OCL}

The Unified Modeling Language (UML) is one of the most frequently used languages to specify and document (software) systems [15]. In particular, class diagrams are very useful to design business applications. In this section we propose to map ClassSheets into UML class diagrams. With this mapping we enable further transformations from spreadsheets to other paradigms (e.g., the object-oriented).

Recall the Salaries ClassSheets model that we presented in Figure 1. In Figure 7 we show it again (Figure 7a) together with its equivalent class diagram (Figure $7 \mathrm{~b}$ ).

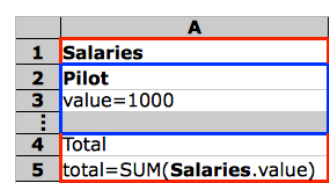

(a) ClassSheets model.

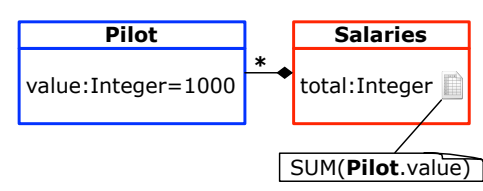

(b) UML class diagram.
Figure 7: Two models for the Salaries sheet.

This example illustrates the similarities between the two representations. The (ClassSheets) Pilot class is represented in UML as a class with the same name and the same attribute, value, of type Integer and initial value 1000. For the Salaries class the transformation is achieved in a similar way, just noticing that total is defined as the sum of the values of the Pilot class. The two classes are connected: Salaries is composed of zero or more Pilot elements.

In the following sections we systematize the transformation of ClassSheets into UML by translating each of the elements that compose the extended ClassSheets language that we have given in Section 3. In fact, we generate specifications within the UML-based Specification Environment (USE): this framework supports UML execution and OCL constraint checking therefore providing analysts, designers and developers with the opportunity to employ model-driven techniques for software production [8].

\subsection{Mapping ClassSheets Into UML Classes}

The mapping from ClassSheets to UML is divided into two phases: firstly, UML classes are generated; secondly, the associations between the generated classes are inferred. In this section we present the first transformation encoded in function $\mathcal{T}$, which uses some auxiliary functions. The first of these functions is getType: it receives a formula $f$, which is used to define the value of an attribute $a$ such that $a=f$, and returns a pair whose first component is the type of $a$ and whose second component is its (textual) definition.

Type $=$ String

Definition $=$ String

AttType $=$ Type $\times$ Definition

getType: Fml $\rightarrow$ AttType

getType $\varphi=\left(\right.$ getType $\left._{1} \varphi, \varphi\right)$

getType $(n \cdot a) \quad=$ getType $($ getAttDef $(n \cdot a))$

getType $(\varphi(f, \ldots, f))=\left(\operatorname{getType}_{2}(\varphi(f, \ldots, f)),(\varphi(f, \ldots, f))\right)$

If an attribute is defined by a default value $\varphi$, getType returns the type of $\varphi$ as a string (given by getType $e_{1}$ ) together with $\varphi$ itself. As an example, if we have an attribute definition $a=\varphi$ such as value $=0$, then the result of getType $\varphi$ is (Integer, 0 ).

In case an attribute is defined as a reference to another, the function getAttDef is used to get such attribute definition. The function getType is then recursively applied to retrieve the attribute's type and definition.

Finally, if an attribute is defined as a formula, getType 2 is used to retrieve the formula's type and the formula definition is itself used to complete the result.

Given the simplicity of getType $e_{1}$, getAttDef and getType , $_{\text {, }}$ their definition is not given here, but it can be seen in the implementation ${ }^{3}$.

Next, we introduce function getAtts, that receives a block $b$ and returns the set of attribute definitions that occur in $b$.

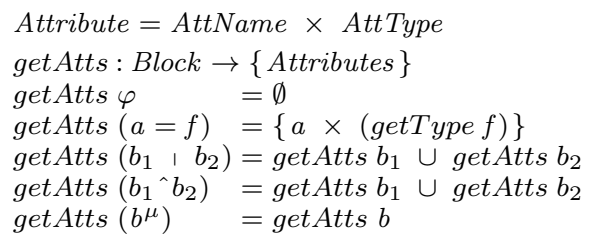

Whenever an attribute definition $a=f$ is found, getAtts creates the singleton set with the name of the attribute being defined, $a$, paired with the result of applying getType to the definition of $a$, i.e., to $f$. In the remaining cases, getAtts simply recurses over the structure of blocks. This function will be used to calculate the attributes of a new UML class.

The next function, called getName, receives the label of a class and returns the string in that label:

$$
\begin{aligned}
& \text { UMLClassName }=\text { String } \\
& \text { getName }: \text { Lab } \rightarrow \text { UMLClassName } \\
& \text { getName }(. n)=n \quad \text { getName }(\underline{n})=n \\
& \text { getName }(\mid n)=n
\end{aligned}
$$

The last auxiliary function, addClass, receives a UML class $c$ (represented by its name and the set of its attributes) and a set $s$ of the classes that have already been mapped from ClassSheets to UML and inserts $c$ in $s$ according to the rule:

${ }^{3}$ That is available in the HaExcel folder of http: //haskell. di.uminho.pt/websvn/ 
$*-*$. The name of this association is the label of the cell class $p$. This association is added to the existing ones and the function recurses over the same input sheet with one difference: the cell class is substituted by an "empty class" so the remaining associations can be found.

The second case occurs when two classes are composed. Here, if parent is equal to the label of the first class, then the function recurses over the second class, but with parent being the parent of the first class. Otherwise, an association between parent and the first class is created. The name of this association is the label of the argument class. The function recurses again over the second class.

In the remaining cases the function recurses over the argument ClassSheets.

As in the previous section, from the output computed by $\mathcal{C}$, we can generate a representation of the associations that can be read by the USE framework. The following function receives an association and returns a string representing it.

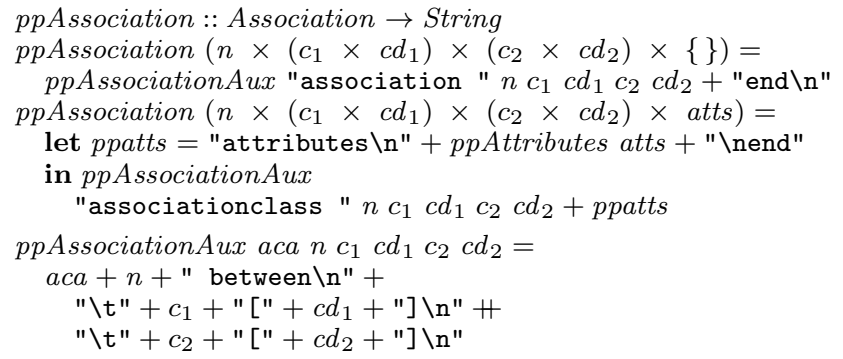

The function has two cases: the first one, with no attributes in the association, generates a regular UML association. For the second case, with attributes, an association class is generated so the attributes can be inserted in the model.

For our example, the associations generated are as follows:

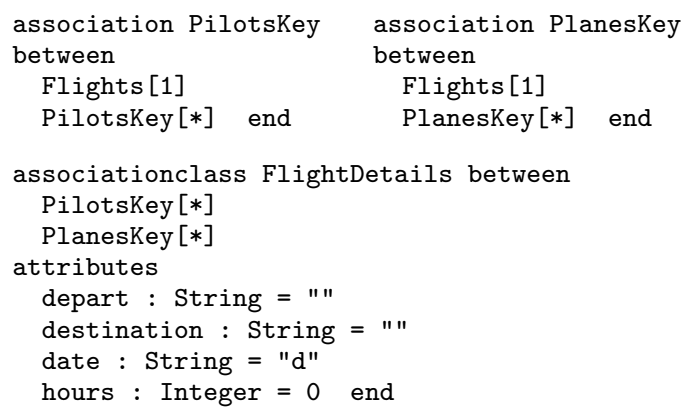

The complete UML class diagram generated by our techniques and drawn by the USE tool is shown in Figure 8:

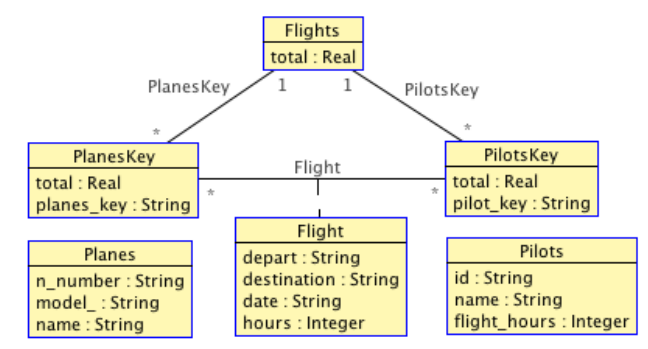

Figure 8: UML class diagram for the flights example.

\subsection{Generating OCL}

In the previous sections we introduced functions to generate UML class diagrams from ClassSheets. But we also need to generate OCL code to guarantee that both representations ensure the same properties. In particular, we need to generate certain restrictions on classes that hold primary and foreign keys. In the case of primary keys, we need to ensure that no value used as such key is repeated. In the case of foreign keys, we need to make sure that all these values correctly point to an existing primary key.

In order to generate the conditions that must invariantly hold for primary key values, we start by defining a function that generates sets of pairs, each one containing the label of the class which the invariant is associated with and a set of blocks that represent the primary key.

$$
\begin{aligned}
& p k: \text { Sheet } \rightarrow\{\text { Lab } \times\{\text { Block }\}\} \\
& p k(l: b)=\{l \times(\text { pkaux b })\} \\
& p k\left(c_{1} \wedge c_{2}\right)=p k c_{1}+p k c_{2} \\
& p k(c \rightarrow)=p k c \\
& p k\left(s_{1} \quad s_{2}\right)=p k s_{1}+p k s_{2}
\end{aligned}
$$

Function $p k$ uses function pkaux to search for all the primary key blocks:

$$
\begin{aligned}
& \text { pkaux }:: \text { Block } \rightarrow\{\text { Block }\} \\
& \text { pkaux }\left(\varphi^{\mu}\right)=\{\varphi\} \\
& \text { pkaux }\left(b_{1} \quad b_{2}\right)=\text { pkaux } b_{1}+\text { pkaux } b_{2} \\
& \text { pkaux }\left(b_{1}{ }^{\wedge} b_{2}\right)=\text { pkaux } b_{1}+\text { pkaux } b_{2} \\
& \text { pkaux }-=\{\}
\end{aligned}
$$

To generate USE compatible constraints, we define a function $p p P K s$ that creates an invariant for each element in the set produced by function $p k$.

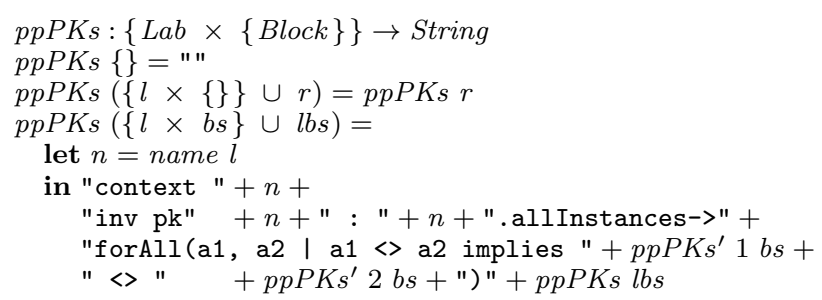

The auxiliary function $p p P K s^{\prime}$ generates a string containing all the blocks. It receives an integer so that it can be reused to generate code for both sides of the inequation on $p p P K s$. For a class with two primary keys "ID" and "name", for example, $p p P K s^{\prime}$ would generate a string such as "a1.ID a1.name".

$$
\begin{aligned}
& \text { ppPKs' }: \text { Integer } \rightarrow\{\text { Block }\} \rightarrow \text { String } \\
& p p P K s^{\prime}-\{\} \quad=" " \\
& p p P K s^{\prime} n(b \cup b s)=" \mathrm{a} "+n+" \cdot "+b+" \text { " }+p p P K s^{\prime} n b s
\end{aligned}
$$

For our running example, the OCL code generated for the Pilots class, and that guarantees the safe use of a primary key value on the pilots identification, is shown next:

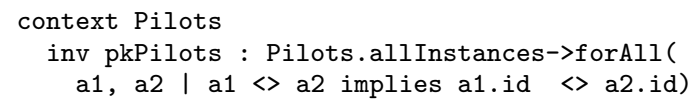

The functions to generate code for foreign key validation are in all similar to the ones shown above. For this reason, we refrain from showing the complete definitions of the functions that implement it. In essence, we define a function that produces lists of pairs, each one containing the label of the class being affected by the constraint and a triple of strings given by an auxiliary function $f k a u x$. The definition of $f k$ for the remaining cases follows the definition of $p k$. 
$f k::$ Sheet $\rightarrow\{$ Lab $\times\{$ String $\times$ String $\times$ String $\}\}$

$f k(l: b)=\{l \times($ fkaux $b)\}$

fkaux $::$ Block $\rightarrow$ String $\times$ String $\times$ String $\}$

fkaux $(k=n \cdot a)=\{k \times n \times a\}$

Each generated element contains the name of the attributes being defined as a foreign key, $k$, the name of the class being referenced, $n$, and the attribute in that class, $a$.

The generated OCL code that implements the foreign plane key validation in the PlanesKey class is as follows.

context planeskey : PlanesKey

inv fkPlanesKey : Planes.allinstances->

exists (a | a.n-number = planeskey.planes_key)

\section{RELATED WORK}

The work presented in this paper has strong connections with [7]. Indeed, in that paper the authors have proposed the ClassSheet modelling language for spreadsheets and used UML to witness the class structure of ClassSheets. Our studies also involve ClassSheets and UML, but several aspects distinguish our paper and [7]. Firstly, we have taken the original ClassSheet language of [7] and extended it with both syntactic constructions and deeper semantics. Namely, we have focused on spreadsheets that can be viewed as relational databases and, for such spreadsheets, our extended modelling language offers more correctness guarantees on the derived spreadsheets than the original one. Secondly, the UML diagrams presented in [7] are given, on concrete examples, just as illustrations of ClassSheet properties. The diagrams shown are quite simple and one can easily see the relation to particular ClassSheets. However, the purpose of [7] was not to obtain them automatically from ClassSheets models. On the contrary, in this paper we have formalised a transformation from the extended ClassSheet language to UML. The generation of OCL to ensure model properties consists of a final distinction between our work and [7].

The derivation of UML class diagrams with OCL constraints from ClassSheets opens a wide range of validation possibilities for spreadsheets. Concrete validation techniques are outside the scope of this paper, but we should point as interesting the combination of our work with some others: [8] offers an animation based approach for the validation of UML models and OCL constraints; [4] provides a validation environment for UML that checks consistency, completeness and dependability requirements. We believe these and other techniques can cooperate to further reduce the still alarming number of errors in spreadsheets.

\section{CONCLUSIONS}

In this paper we have extended ClassSheets with characteristics from relational databases. This means that we are now able of specifying database oriented spreadsheets where it is possible to define primary and foreign keys.

We have also shown how to systematically transform an extended ClassSheet into a UML class diagram (classes, associations and OCL restrictions). UML class diagrams are generated under the notation of the USE framework, and thus we allow for immediate verification of the spreadsheet model. This framework allows one to create instances of the given UML model and verify the OCL constraints on those objects. Moreover, it is also possible to define pre and postconditions on operations. From this, it must also be possible to generate spreadsheet macros/formulas to ensure the constraints. This is an aspect that we have not fully explored yet, but that we plan to do in the future.

We have implemented our transformation in the HASKELL programming language under the HAExCEL framework. The tests we have run show, for example, that we generate the same UML models shown in [7].

\section{REFERENCES}

[1] R. Abraham, M. Erwig, S. Kollmansberger, and E. Seifert. Visual specifications of correct spreadsheets. In VLHCC '05: Procs. of the 2005 IEEE Symposium on Visual Languages and Human-Centric Computing, pages 189-196. IEEE Computer Society, 2005.

[2] J. Bézivin. Model Driven Engineering: An Emerging Technical Space. In R. Lämmel, J. Saraiva, and J. Visser, editors, GTTSE 2005, volume 4143 of LNCS, pages 36-64. Springer, 2006.

[3] C. Chambers and C. Scaffidi. Struggling to excel: A field study of challenges faced by spreadsheet users. In 2010 IEEE Symp. on Visual Languages and Human-Centric Computing, pages 187-194, Los Alamitos, CA, USA, 2010. IEEE Computer Society.

[4] G. Csertán, G. Huszerl, I. Majzik, Z. Pap, A. Pataricza, and D. Varró. VIATRA - Visual Automated Transformations for Formal Verification and Validation of UML models. In Automated Software Engineering, pages 267-270, 2002.

[5] J. Cunha, J. Mendes, J. P. Fernandes, and J. Saraiva. Embedding and evolution of spreadsheet models in spreadsheet systems. In Procs. of the IEEE Symposium on Visual Languages and Human-Centric Computing, 2011.

[6] J. Cunha, J. Saraiva, and J. Visser. Discovery-based edit assistance for spreadsheets. In Proc. of the 2009 IEEE Symposium on Visual Languages and Human-Centric Computing, pages 233-237, Washington, DC, USA, 2009. IEEE Computer Society.

[7] G. Engels and M. Erwig. ClassSheets: Automatic generation of spreadsheet applications from object-oriented specifications. In ASE '05: Proceedings of the 20th IEEE/ACM International Conference on Automated Software Engineering, pages 124-133, New York, NY, USA, 2005. ACM.

[8] M. Gogolla, F. Büttner, and M. Richters. Use: A UML-based specification environment for validating UML and OCL. Sci. Comput. Prog., 69:27-34, 2007.

[9] F. Hermans, M. Pinzger, and A. van Deursen. Automatically extracting class diagrams from spreadsheets. In Proc. of the 24th European Conference on Object-Oriented Programming, pages 52-75, Berlin, Heidelberg, 2010. Springer-Verlag.

[10] D. Maier. The Theory of Relational Databases. Computer Science Press, 1983.

[11] OOoAuthors. Getting Started with OpenOffice.org 3. CreateSpace, 2010.

[12] R. R. Panko. Spreadsheet errors: What we know. What we think we can do. Proceedings of the Spreadsheet Risk Symposium, European Spreadsheet Risks Interest Group (EuSpRIG), July 2000.

[13] S. G. Powell and K. R. Baker. The Art of Modeling with Spreadsheets. John Wiley \& Sons, Inc., New 
York, NY, USA, 2003.

[14] K. Rajalingham, D. Chadwick, and B. Knight.

Classification of spreadsheet errors. European

Spreadsheet Risks Interest Group (EuSpRIG), 2001.

[15] J. Rumbaugh, I. Jacobson, and G. Booch. Unified Modeling Language Reference Manual, The (2nd Edition). Pearson Higher Education, 2004. 